\title{
Post-buckling behaviour of carbon-nanotube-reinforced nanocomposite plate
}

\author{
ASHISH SRIVASTAVA* and DINESH KUMAR \\ Department of Mechanical Engineering, Malaviya National Institute of Technology, Jaipur 302017, India \\ e-mail: ashish.memech@gmail.com; dkumar.mech@mnit.ac.in
}

MS received 5 January 2016; revised 17 April 2016; accepted 14 June 2016

\begin{abstract}
The aim of the present paper is to investigate the buckling and post-buckling behaviour of nanocomposite plate having randomly oriented carbon nanotubes (CNTs) reinforced in magnesium $(\mathrm{Mg})$ under uni-axial compression. The effect of non-bonded interaction at the interface between CNT and matrix is considered through a cohesive zone model, used to predict the elastic property of the interphase, while evaluating the elastic properties of the nanocomposite using a representative volume element. A special purpose program based on finite-element formulation is developed to study the buckling and post-buckling behaviour of nanocomposite plate. The formulation is based on first-order shear deformation theory in conjunction with geometrical non-linearity as per von Karman's assumptions. A parametric study is conducted to investigate the effects of interphase between CNT and matrix, short-CNT and long-CNT reinforcements and boundary conditions on buckling and post-buckling response of nanocomposite plate. It is found that imperfect bonding between CNT and $\mathrm{Mg}$ results in the loss of buckling and post-buckling strength, as compared with perfect bonding, of CNT-Mg nanocomposite plate. It is also concluded that buckling and post-buckling strength is higher for long-CNT-reinforced nanocomposite plate than that of short-CNT reinforcement, irrespective of bonding between CNT and matrix material.
\end{abstract}

Keywords. Carbon nanotube (CNT); representative volume element (RVE); cohesive zone model; finiteelement method (FEM); post-buckling.

\section{Introduction}

Carbon nanotubes (CNTs) are 1D cylindrical structure of hexagonally arranged carbon atoms having elastic modulus of $1 \mathrm{TPa}$ and tensile strength of over $150 \mathrm{GPa}$ [1-3]. These CNTs are considered as a potential candidate for reinforcement to form advanced structural materials, if good interfacial bonding between CNT and matrix material and proper dispersion of the CNTs in matrix material are obtained [4]. In a study made by Esawi et al [5], enhancements of tensile strength and stiffness of CNT-reinforced aluminium nanocomposite were reported up to $50 \%$ and $23 \%$, respectively, in comparison with pure aluminium.

The high strength and the stiffness of the CNT are futile, if the applied load on the nanocomposite is not transferred to these CNTs through interface. Therefore a strong interface between the CNT and matrix material is necessary for effective load transfer [6]. The strength of interface is strongly influenced by the structure and type of the matrix material [7]. Zhang et al [8] established that in case of polyethylene nanocomposite, the interphase zone around

*For correspondence the CNT/GS has higher strength and density than those of the matrix material.

To study the behaviour of interface, the molecular dynamics (MD) and the finite-element method (FEM) based on continuum mechanics have been widely used by the researchers $[9,10]$ owing to their specific capabilities to solve the problems at nano- and micro-scales. Irrespective of the capabilities of MD to capture the effect of force field between the atoms, and modelling environment (like pressure, temperature and system total energy) more accurately than FEM, it is limited to small length scales (i.e., hardly a few thousand atoms) and time scales (i.e., in the range of a few pico-seconds), which makes it computationally inefficient for the study of advanced structures having large dimensions. Therefore FEM plays a crucial role in minimizing the computational cost of simulation, and many researchers have utilized its capabilities to predict the mechanical properties of nanocomposites at nano- and micro-scales using the method of representative volume element (RVE); for instance, Liu and Chen [11, 12] and Joshi et al [13]. To simulate the effect of van der Waals (i.e., vdW) forces between CNT and matrix material using FEM, different methodologies have been devised by many researchers. For instance, Shokrieh and Rafiee [14] 
modelled the interphase zone between single-wall CNT (SWCNT) and polymer matrix using spring elements between the CNT and matrix material, and Joshi and Upadhyay [10, 15] developed the interface zone using spring elements between the multi-wall CNT (MWCNT) and matrix material to predict the mechanical properties of nano-composite. To reduce the computational cost, an equivalent continuum cylindrical interphase model was also developed by Tsai et al [16] and Herasati et al [17] to mimic the effect of interphase. Shokrieh and Rafiee [18] proposed an equivalent long fibre consisting of CNT and interphase region to carry out micromechanical studies. Very recently, Rafiee and Pourazizi [19] studied the effect of functionalization of CNT on the elastic behaviour of nanocomposite by modelling the $\mathrm{vdW}$ interaction and covalent bonds between CNT and matrix, and revealed that chemical functionalization will reduce the Young's modulus of the RVE at microscale in comparison with the RVE containing CNT without functionalization.

Knowing the fact that interphase region has a significant effect on the effective mechanical properties of nanocomposite [20], it is required to discuss its mechanical behaviour on a proper theoretical background. A generalized equation of interphase potential based on $\mathrm{vdW}$ interaction between CNT and polymer matrix was given by Jiang et al [21]. Tan et al [22] incorporated the non-linear cohesive law, derived by Jiang et al [21], for CNT/polymer interfaces in the study of the macroscopic behaviour of CNTreinforced composites, and concluded that at small strain CNTs improve the mechanical behaviour of the composite, but at large strain such improvements disappear because of complete debonding of CNTs from matrix. The same cohesive energy model of interphase zone was used by Zhao et al [23] to investigate the effects of size, spacing and crossing angles of CNT, graphene and substrates on the cohesive energy of interphase zone. Further, Zhang et al [24] modelled a functionally graded variation of interphase (FGVI) using the same cohesive zone model of Jiang et al [21] between CNT and polymer matrix and studied the effect of FGVI on composite stiffness and cohesive strength.

The above-mentioned studies have revealed that it would be important and useful to explore the potential applications of CNT-reinforced nano-composites in actual structural components, such as plates and shells. One of the most interesting characteristics of thin-plate-like structural components is to withstand larger in-plane (compression and/or shear) load after reaching buckling/bifurcation point in the post-buckling regime. Different buckling and postbuckling studies of CNT-reinforced plates are available in the literature. Arani et al [25] used the Mori-Tanaka method to predict the effective elastic modulus of randomly oriented nanocomposite to study the buckling behaviour of CNT-reinforced laminated composite plates. The MoriTanaka method was also used by Jafari Mehrabadi et al [26] and Lei et al [27] for the stability analysis of functionally graded CNT-reinforced plates (FGCRP). Shen and Zhang [28] predicted the thermal buckling and postbuckling behaviour of FGCRP and reported enhanced buckling temperature and post-buckling strength of the plate by functionally graded reinforcement of CNT. Shen and Zhu [29] studied the post-buckling strength of a plate, sandwiched by CNT-reinforced composite face sheets, resting on elastic foundations and revealed that the foundation stiffness, the temperature changes, the nanotube volume fraction of face sheet and the core-to-face sheet thickness ratio have significant effects on the compressive buckling load and post-buckling behaviour of the sandwich plate, whereas this effect on the thermal post-buckling behaviour is less pronounced for the same sandwich plate.

From the available literature, it is found that the interphase region between a nanofiller and matrix material significantly influences the effective mechanical properties of the nanocomposite, which in turn would affect significantly the buckling and post-buckling behaviour of the resulting nanocomposite plate at macro-scale. In the present study, interphase region between CNT and matrix is modelled using vdW-cohesive zone model as introduced by Jiang et al [21] to mechanically characterize the interface between the CNT and matrix. The method of RVE is used to evaluate the stiffness properties of nanocomposite reinforced with randomly oriented CNTs. The predicted stiffness properties of the nanocomposite are utilized to investigate the buckling and post-buckling behaviour of nanocomposite plate. A special purpose program based on non-linear finite-element formulation is developed to carry out the study. The non-linear finite-element formulation is based on the first-order shear deformation theory and von Karman's assumptions, and the non-linear finite-element algebraic equations are solved using the Newton-Rapshon method.

\section{Elastic modulus of interphase region}

The non-bonded interactions between the CNT and matrix materials are described by the van-der-Waals-force-based cohesive zone model established by Jiang et al [21], for predicting the cohesive stresses (tensile and shear) between CNT and matrix materials. The same model has been used in the present study to predict the linear elastic behaviour of interphase zone to predict its stiffness property. The equations for the cohesive energy (per unit length of the CNT) of interphase zone between the CNT and matrix are described as follows:

$$
\phi=\frac{2 \pi^{2}}{3} \rho_{m} \rho_{\mathrm{C}} \sigma_{I}^{3} \varepsilon_{I}\left[2 R\left(\frac{2 \sigma_{I}^{9}}{15 r^{9}}-\frac{\sigma_{I}^{3}}{r^{3}}\right)+\frac{3}{2} \sigma_{I}\left(\frac{\sigma_{I}^{8}}{10 r^{8}}-\frac{\sigma_{I}^{2}}{r^{2}}\right)\right]
$$

where area density $\left(\rho_{\mathrm{C}}\right)$ of the CNT is the number of carbon atoms per unit area of CNT surface and is given by 
$\rho_{\mathrm{C}}=\frac{4}{3 \sqrt{3} l_{\mathrm{c}}}, l_{\mathrm{C}}$ being the equilibrium bond length between carbon atoms prior to deformation, $\rho_{m}$ is the volume density of matrix material (i.e., number of matrix-material atoms per unit volume), $r$ represents the distance between the inner surface of matrix material and the outer surface of CNT, $R$ is the outer radius of CNT, and $\sigma_{I}$ and $\varepsilon_{I}$ are the intermolecular potential parameters.

$\sigma_{I}$ and $\varepsilon_{I}$ for the interphase between CNT atoms and the atoms of matrix materials can be calculated by applying the widely used Lorentz-Berthelot (LB) mixing rule [30, 31]. This rule is used for estimating intermolecular potential parameters between the pairs of non-identical molecules from those between the pairs of corresponding identical molecules, as follows:

$$
\begin{aligned}
\sigma_{I} & =\frac{\sigma_{\mathrm{C}}+\sigma_{m}}{2} \\
\varepsilon_{I} & =\sqrt{\varepsilon_{\mathrm{C}} \varepsilon_{m}} .
\end{aligned}
$$

Interphase potential as given by Eq. (1) is considered as the total strain energy of the assumed isotropic interphase material, and the stiffness property of this isotropic interphase material can be predicted using the following equation, as used by Sears and Batra [32]:

$$
E=\frac{\partial^{2} \phi}{\partial^{2} S}
$$

where $S$ is the strain at a distance $r$ obtained as

$$
S=\frac{r-h_{0}}{h_{0}}
$$

wherein the $h_{0}$ represents the equilibrium distance (also called the thickness of interphase zone) between CNT and matrix material and determined by minimizing the interphase potential (i.e., $\partial \phi / \partial r=0$ ) as

$$
h_{0}=0.8584 \sigma_{I} .
$$

The elastic modulus of the interphase is evaluated for the equilibrium position (i.e., $r=h_{0}$ ), and the expression, obtained from Eq. (4), for the Young's modulus of the interphase material between CNT and matrix is given as

$E=\frac{2}{3} h_{0} \pi^{2} \rho_{m} \rho_{C N T} \sigma_{I} \varepsilon_{I}\left[24 R\left\{\left(\frac{\sigma_{I}}{r}\right)^{11}-\left(\frac{\sigma_{I}}{r}\right)^{5}\right\}+9 \sigma_{I}\left\{\frac{6}{5}\left(\frac{\sigma_{I}}{r}\right)^{10}-\left(\frac{\sigma_{I}}{r}\right)^{4}\right\}\right]$.

It is necessary to mention here that $E$ obtained from Eq. (7) for the interphase between CNT and matrix material will have unit of force per unit length, and hence needs to be divided by average circumference [i.e., by $2 \pi\left(R+\frac{h_{0}}{2}\right)$, obtained as average of outer circumference of CNT (i.e., $2 \pi R$ ) and inner circumference of matrix material (i.e., $\left.\left.2 \pi\left(R+h_{0}\right)\right)\right]$.

\section{Homogenization of randomly oriented CNT composite}

\subsection{Generation of randomly oriented CNT composite RVE model}

The common approach to evaluate the macroscopic properties of CNT-reinforced nanocomposite is to create an RVE, also called unit cell, which captures the major features of the underlying CNT reinforced in matrix. A cubic RVE is modelled using the livelink between COMSOL MultiPhysics and MATLAB, in which the spatial coordinates of the centre of each CNT are predefined by generating an array of CNTs in such a way that the outermost edges of the CNTs should not cross the RVE boundary. Then, in order to get the isotropic behaviour of the nanocomposite, periodically positioned 27 CNTs are oriented randomly in 3D by generating a range of uniformly distributed pseudo-random integers between $0^{\circ}$ and $180^{\circ}$, as shown in figure 1 . This RVE with periodic boundary conditions, as given by Sun and Vaidya [33], is used to predict the effective elastic constants of the nanocomposite through finite-element analysis using COMSOL MultiPhysics. The RVE was meshed with tetrahedron elements using physicscontrolled feature of the software.

\subsection{Numerical homogenization in random media}

At the nanoscale, the RVE having a matrix reinforced with CNT is actually a heterogeneous composite medium, which is to be used for evaluating effective (i.e., average) material properties of the nanocomposite that is considered to be homogeneous at larger scales (i.e., micro- and mesoscales). Therefore, it is required to use a homogenization technique to find a globally homogeneous medium equivalent to the original heterogeneous composite medium at

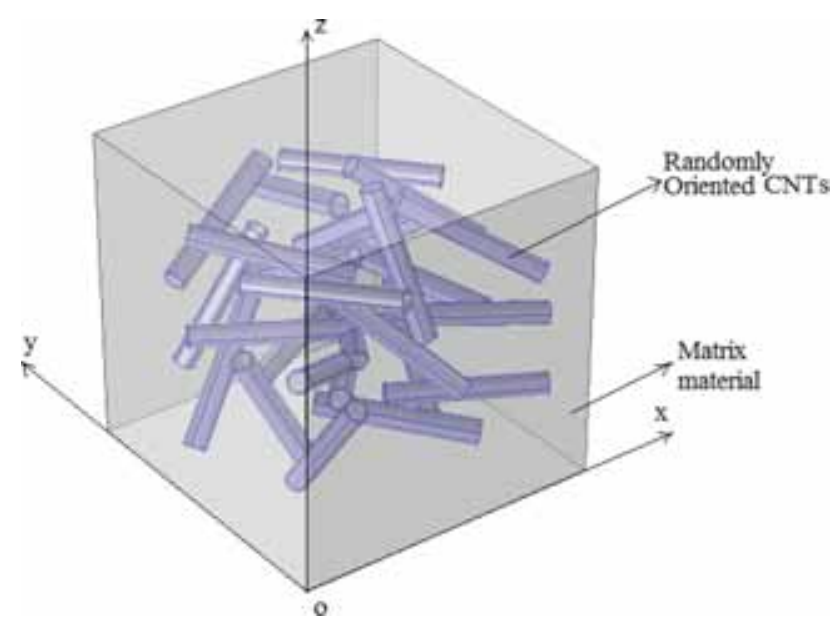

Figure 1. Composites RVE reinforced with randomly oriented and periodically positioned CNTs. 
nanoscale and to reduce the non-homogeneous stress and strain fields within the heterogeneous material obtained from the finite-element analysis of RVE to the volumeaveraged stress and strain. Following paragraphs contain a homogenization procedure to determine the effective moduli that describe the 'average' material properties of the actual heterogeneous nanocomposite.

Individual phases have isotropic material properties, and it is assumed that the constitutive law in the matrix and the reinforcement is given by the following generalized Hooke's Law:

$$
\sigma_{i j}=C_{i j k l} \varepsilon_{k l}, \quad i, j, k, l=1,2,3
$$

where $\sigma_{i j}, \varepsilon_{k l}$ and $C_{i j k l}$ are the coefficients of the stress tensor, the linear strain tensor and the stiffness tensor, respectively.

The FEM analysis of the RVE would yield the abovementioned stress and strain fields within the heterogeneous material. The effective (i.e., averaged) stiffness coefficients of nanocomposite (at micro- or macro-scale) can be calculated from

$$
\bar{\sigma}_{i j}=C_{i j k l}^{e} \bar{\varepsilon}_{k l}
$$

where $C_{i j k l}^{e}$ refers to the effective stiffness tensor, and $\bar{\sigma}_{i j}$ and $\bar{\varepsilon}_{k l}$ are, respectively, the volume-averaged stress and strain tensors calculated over the volume of the RVE using the following volumetric integral expressions:

$$
\begin{aligned}
& \bar{\sigma}_{i j}=\frac{1}{V_{R V E}} \int_{V_{R V E}} \sigma_{i j}(x, y, z) d V \\
& \bar{\varepsilon}_{k l}=\frac{1}{V_{R V E}} \int_{V_{R V E}} \varepsilon_{k l}(x, y, z) d V
\end{aligned}
$$

where $\bar{\sigma}_{i j}$ and $\bar{\varepsilon}_{k l}$ are the volume-averaged stress and strain tensors calculated over the volume of the RVE. $V_{R V E}$ represents the volume of RVE.

Further, stiffness properties of resultant nanocomposite are calculated from the following equation:

$$
\bar{\sigma}_{i j}=C_{i j k l}^{e} \bar{\varepsilon}_{k l}
$$

where $C_{i j k l}^{e}$ refers to the effective stiffness tensor of the nanocomposite. After calculating the volume-averaged stress and strain components, the relevant nanocomposite moduli (i.e., effective/averaged stiffness coefficients) can be obtained from average stresses and average strains. For three pure normal strain states, the stiffness tensors can be written as

$$
C_{i j k l}^{e}=\frac{\bar{\sigma}_{i j}}{\bar{\varepsilon}_{k l}} ; \quad \text { with } i=j=k=l \text {. }
$$

\section{Micromechanics model}

In this section the semi-empirical approach used for the validation of FEA-based procedure discussed in section 3 is described. Under the assumption of perfect bonding between the reinforcement and matrix, Tsai and Pagano [34] proposed a semi-empirical model to predict the effective stiffness properties of RVE having randomly oriented fibres. This model, widely used by many authors (for instance, [35, 36], is applied in the present study to predict the effective stiffness properties of nanocomposite reinforced with randomly oriented CNTs. The Tsai-Pagano model is given by the following equations:

$$
\begin{aligned}
E & =\frac{3}{8} E_{1}+\frac{5}{8} E_{2} \\
G & =\frac{1}{8} E_{1}+\frac{1}{4} E_{2}
\end{aligned}
$$

where $E$ and $G$ represent the Young's and shear moduli of the resulting isotropic nanocomposite, respectively; $E_{1}$ and $E_{2}$ are the longitudinal and transverse Young's modulus of the single-CNT-reinforced RVE.

$E_{1}$ and $E_{2}$, required in Eqs. (14) and (15), of the RVE can be evaluated using the FEM-based homogenization method as discussed in section 3.2, and the method will be regarded as FEM-based Tsai-Pagano method in the current study.

Moreover, $E_{1}$ and $E_{2}$ can also be estimated analytically through the Halphin-Tsai method as given by following equations:

$$
\begin{gathered}
\frac{E_{1}}{E_{m}}=\frac{1+\xi \eta v_{f}}{1-\eta v_{f}} \quad \text { where } \eta=\frac{\frac{E_{f}}{E_{m}}-1}{\frac{E_{f}}{E_{m}}+\xi} \text { and } \xi=\frac{2 L}{d} \\
\frac{E_{2}}{E_{m}}=\frac{1+\xi \eta v_{f}}{1-\eta v_{f}} \quad \text { where } \eta=\frac{\frac{E_{f}}{E_{m}}-1}{\frac{E_{f}}{E_{m}}+\xi} \text { and } \xi=2
\end{gathered}
$$

where $\xi$ is the reinforcing factor, which depends upon reinforcement geometry, packing geometry and loading condition. For a reinforcement of circular cross-section in a packing geometry of square array, $\xi=2$. Subscripts ' $m$ ' and ' $f$ ' correspond to the matrix and the reinforcement (i.e., CNT), respectively. The volume fraction $v_{f}$ of the reinforcement in the RVE is given by

$$
v_{f}=\frac{V_{C N T}}{V_{R V E}}=\frac{n \pi\left(r_{o}^{2}-r_{i}^{2}\right) L_{C N T}}{\left(a^{2}-n \pi r_{i}^{2} L_{C N T}\right)}
$$

where $r_{o}$ and $r_{i}$ are the and external and internal radii of the CNT, respectively, $a$ is the side of square RVE, $L_{C N T}$ and $L$ stand for the lengths of CNT and RVE, respectively, and, $n$ is the number of CNTs. 


\section{Present study}

The post-buckling behaviour of a nanocomposite plate is studied in two steps. In the first step, the stiffness properties of RVE reinforced with randomly oriented CNTs are evaluated by considering the effect of interphase between CNT and matrix material. In the second step, the obtained stiffness properties of nanocomposite are utilized to further study the post-buckling behaviour of nanocomposite plate using finite-element analysis.

To predict the stiffness properties of CNT-reinforced nanocomposite, multi-CNT-reinforced RVE (see figure 1) and two single-CNT RVEs: CNT through RVE and CNT inside RVE, as shown in figure 2 , are considered. CNT through RVE (see figure 2a) is the case of relatively long CNTs having large aspect ratio and therefore a segment of it is modelled using an RVE, as utilized by many researchers, e.g., Liu and Chen [11], Joshi et al [37] and
Kumar and Srinivas [38]. Similarly, to study short-CNTreinforced nanocomposite, CNT inside RVE (see figure $2 \mathrm{~b}$ ) having RVE length double as that of CNT length, also considered by Liu and Chen [11] and Joshi et al [37], is considered in the current study. For the case of single-CNTreinforced RVE, the stiffness properties of the nanocomposite are evaluated using the FEM-based Tsai-Pagano method discussed in section 4.

Based on the past studies, e.g., Kumar and Srinivas [38], Liu and Chen [11] and Joshi et al [37], the dimensions and elastic properties of CNT considered in the present study are as follows:

length $L_{C N T}=50 \mathrm{~nm}$; thickness of CNT $t=0.4 \mathrm{~nm}$;

Young modulus $E_{C N T}=1$

$\mathrm{TPa}$; Poisson's ratio $v=0.3$; outer radius $r_{o}=3.6 \mathrm{~nm}$ and inner radius $r_{i}=3.2 \mathrm{~nm}$.
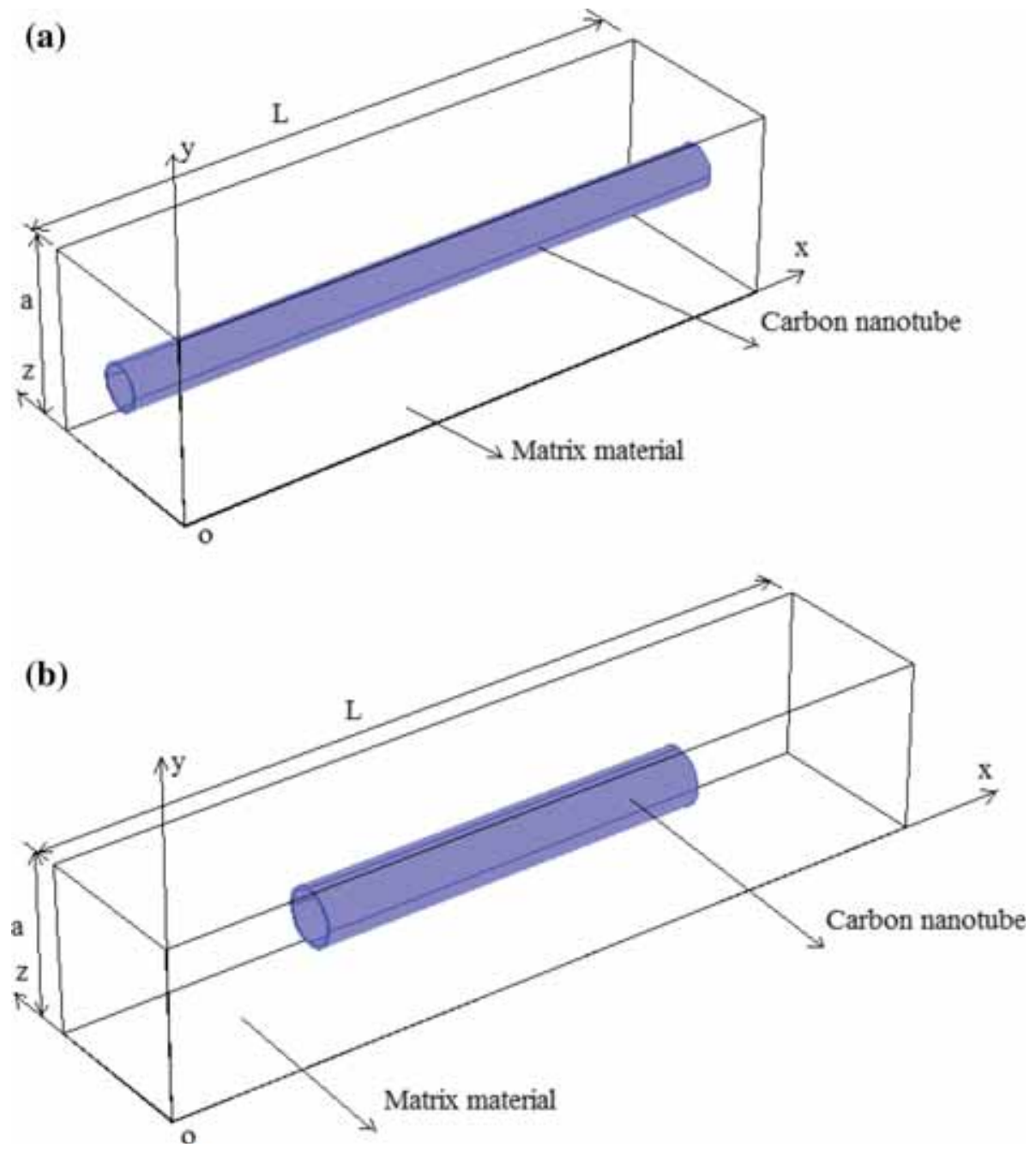

Figure 2. (a) CNT through RVE and (b) CNT inside RVE. 


\subsection{Evaluation of elastic properties of nanocomposite reinforced with $3 D$-rotated CNTs}

5.1a Validation: In this section, different approaches to evaluate the elastic modulus of isotropic nanocomposite (with $v_{f}=1 \%$ ) having randomly oriented CNTs reinforced in different matrix materials are discussed to validate and judge for an efficient approach in terms of computational cost and accuracy for a nanocomposite with higher volume fraction (e.g., 4\% as used in the subsequent study of present paper). The four approaches are (i) FEM-based 3D RVE reinforced with randomly oriented CNTs (i.e., multi-CNT model), (ii) semi-empirical analytical Tsai-Pagano method, (iii) FEM-based Tsai-Pagano using single-CNT inside RVE and (iv) FEM-based Tsai-Pagano using single CNT through RVE. The different matrix materials considered are: $\quad \operatorname{poly}\{(\mathrm{m}$-phenylene-vinylene $)-c o-[(2,5$-dioctoxy-pphenylene)vinylene]\} (PmPV), magnesium (Mg), copper $(\mathrm{Cu})$ and steel.

It is to be mentioned here that at macro-scale actual CNTs in the real nanocomposite will be randomly oriented and hence would possess isotropic property. Therefore, an RVE reinforced with 3D randomly oriented CNTs will provide more realistic value of elastic modulus for such isotropic material. But this approach would be computationally more expensive-it would be directly proportional to the number of CNTs used in the RVE, which in turn depends upon the volume fraction of CNT (i.e., $v_{f}$ ). The values obtained for elastic modulus of the composite reinforced by randomly oriented CNTs using above-mentioned approaches are provided in table 1. It can be seen from table 1 that the elastic moduli of nanocomposites obtained through the FEM based multi-CNT model and FEM based Tsai-Pagano model for single-CNT inside RVE are in good agreement with that of analytical Tsai-Pagano model. It is found that in comparison to analytical Tsai-Pagano model a maximum deviation of $8 \%$ and $14 \%$ in elastic modulus is obtained in the case of PmPV matrix, respectively, for FEM based multi-CNT model and FEM based Tsai-Pagano model for single-CNT inside RVE, while this deviation is found to be only $0.3 \%$ and $0.5 \%$ in the case of steel matrix. This large deviation in the elastic modulus for soft PmPV can be attributed to the fact that the enhancement in stiffness properties of nanocomposite is more prevalent for soft matrix material like PmPV than that for stiff matrix like steel, also reported by Joshi and Upadhyay [15].

It can also be noted from table 1 that FEM-based TsaiPagano using single-CNT through RVE approach overestimates the elastic modulus of nanocomposite reinforced with randomly oriented CNTs, as compared with other three approaches. This can be explained in following texts. In the case of multi-CNT model, CNTs do not cross the RVE boundary. Therefore, after the application of periodic boundary conditions, the length of CNTs is finite, thus resembling the case of short-CNT-reinforced nanocomposite. Further, the semi-empirical analytical Tsai-Pagano method utilizes the Halphin-Tsai method to predict the stiffness properties of nanocomposite, which has been widely used to predict the elastic properties of conventional composites having finite (i.e., short) reinforcement (see [39]. At the same time, single-CNT-inside-RVE model also represents the case of short-CNT-reinforced nanocomposite, whereas single-CNT through RVE represents the case of long-CNT-reinforced nanocomposite, as mentioned by Liu and Chen [11] and Joshi et al [37].

It is necessary to mention here that based on the computational cost and accuracy, FEM-based Tsai-Pagano models: using single CNT inside RVE and single CNT through RVE, are used in the current study to evaluate the elastic modulus of nanocomposite reinforced with randomly oriented CNTs (for volume fraction of $v_{f}=4 \%$ ), taking into account the interface between CNT and matrix material. It is necessary to mention here that the reason for choosing the low volume fraction of CNT (equal to 4\%) is that in real practice high volume fraction of CNT would cause agglomeration of CNTs and hence, to avoid this problem, CNT volume fraction is taken low, as used by many authors, e.g., Liu and Chen [11], Joshi et al [37], Hu et al [40] and Joshi and Upadhyay [10].

Table 1. Comparison of Young's modulus of nanocomposite reinforced with randomly oriented CNTs (with $v_{f}=1 \%$ ) having perfect bonding between CNT and matrix material.

\begin{tabular}{lcccc}
\hline & \multicolumn{3}{c}{ Young's modulus (GPa) } \\
\cline { 2 - 5 } $\begin{array}{l}\text { Matrix } \\
\text { material }\end{array}$ & $\begin{array}{c}\text { FEM-based multi- } \\
\text { CNT model }\end{array}$ & $\begin{array}{c}\text { Tsai-Pagano } \\
\text { (analytical) }\end{array}$ & $\begin{array}{c}\text { FEM-based Tsai-Pagano (single } \\
\text { CNT inside RVE) }\end{array}$ & $\begin{array}{c}\text { FEM-based Tsai-Pagano (single } \\
\text { CNT through RVE) }\end{array}$ \\
\hline PmPV (3.51 & 4.0952 & 3.7638 & 4.2867 & 7.6518 \\
$\quad \mathrm{GPa})^{*}$ & 48.0508 & 47.2311 & 48.9122 & 50.18289 \\
$\mathrm{Mg}(45 \mathrm{GPa})$ & 81.9249 & 81.1117 & 82.7149 & 83.57358 \\
$\mathrm{Au}(78 \mathrm{GPa})$ & 134.6879 & 133.9525 & 135.3077 & 135.8984 \\
$\mathrm{Cu}(130 \mathrm{GPa})$ & 205.1319 & 204.5249 & 205.5776 & 206.0082 \\
Steel & & & \\
$\quad(200 \mathrm{GPa})$ & & & & \\
\hline
\end{tabular}

* Elastic modulus of matrix material. 
5.1b Elastic properties of nanocomposite considering the interface between CNT and matrix: In this section, elastic properties of nanocomposite reinforced with randomly oriented CNTs, with $4 \%$ volume fraction of CNT, taking into account the interface between CNT and matrix material, are evaluated using FEM-based Tsai-Pagano models having single CNT inside RVE and single CNT through RVE.

While modelling the RVE (as shown in figure 3), initially the thickness of interphase zone between CNT and $\mathrm{Mg}$ (i.e., equilibrium distance $h_{0}$ ) is evaluated using Eq. (6) and then sides of the RVE are computed using the following equation:

$$
V_{f}=\frac{\pi\left(r_{o}^{2}-r_{i}^{2}\right) L_{C N T}}{a^{2} L-\pi r_{i}^{2} L_{C N T}-\pi\left\{\left(r_{o}+h_{0}\right)^{2}-r_{o}^{2}\right\} L_{C N T}}
$$

The elastic modulus of the non-bonded interphase zone is computed using Eq. (7), for which the required values of $\mathrm{L}_{-}$

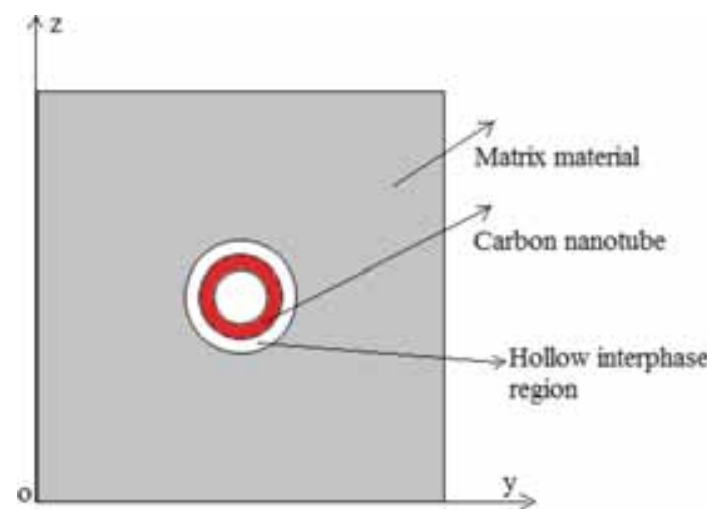

Figure 3. CNT-reinforced RVE having vacant interphase zone.

Table 2. Potential parameters, density and atomic mass unit of magnesium $(\mathrm{Mg})$.

\begin{tabular}{|c|c|c|c|}
\hline$\sigma\left(\mathrm{A}^{0}\right)$ & $\varepsilon(\mathrm{eV})^{*}$ & Density $\left(\mathrm{g} / \mathrm{cm}^{3}\right)$ & Atomic mass unit $(\mathrm{amu}) * *$ \\
\hline 3.0030 & 0.0012 & 1.7380 & 24.3050 \\
\hline
\end{tabular}

J potential parameters i.e., vdW radius $\sigma$ and bond energy $\varepsilon$ [41], and density and atomic mass unit [42] of Mg matrix are given in table 2 . The values of mass density (i.e., $\rho_{m}$ ) of magnesium matrix materials can be evaluated by the ratio of matrix mass density to the mass of per unit atom of the matrix, as given in table 2. For carbon atoms in CNT, the values of bond energy and vdW radius between a pair of carbon atoms are taken as $\varepsilon_{\mathrm{C}}=0.002390 \mathrm{eV}$ and $\sigma_{\mathrm{C}}=0.3415 \mathrm{~nm}$, respectively, and the value of the area density of carbon atoms in CNT is taken as $3.8177 \times$ $10^{19} \mathrm{~m}^{-2}$ [43]. These vdW radii and bond energies of $\mathrm{Mg}$ and $\mathrm{C}$ atoms are utilized to evaluate the $\mathrm{vdW}$ radius and bond energy for interphase region (i.e., $\sigma_{I}$ and $\varepsilon_{I}$ ) using the LB mixing rule [as specified by Eqs. (2) and (3) of section 2].

Using the above-described procedure, the elastic modulus of the hypothetical interphase zone material and the thickness of the interphase zone are calculated as $3.1885 \mathrm{GPa}$ and $0.2755 \mathrm{~nm}$, respectively. This interphase material is filled into the vacant space between CNT and matrix material to study the effect of interphase zone on the elastic properties of nanocomposite.

The deformations and stresses of the resulting RVE are computed for the normal loading in longitudinal and transverse directions to evaluate $E_{1}$ and $E_{2}$ using Eqs. (8)(13), and subsequently using the same for determining $E$ and $G$ of the final isotropic nanocomposite using Eqs. (14) and (15).

A comparison is made between the elastic properties of perfectly and imperfectly bonded short-CNT- and longCNT-reinforced nanocomposites and the results are listed in table 3.

The values of Young's and shear moduli for the imperfectly bonded nanocomposite for both kinds of RVEs (i.e., CNT inside RVE and through RVE) are found to be reduced by $10-11 \%$ as compared with perfectly bonded nanocomposite. This loss in elastic properties can be explained by the von-Mises stress distribution for a typical short-CNT-reinforced RVE, as shown in figure 4, under axial loading condition. It can be noted from figure 4 that the weak interphase between CNT and matrix material carries very less load and it would be a weak link between the matrix and CNT while transmitting load from matrix to CNT. Therefore, the effective load transmitted to the reinforcement would be reduced in the case of imperfect

Table 3. Stiffness properties of CNT-Mg nanocomposite (with $v_{f}=4 \%$ ) using single-CNT (inside and through) RVE model using FEM-based Tsai-Pagano method.

\begin{tabular}{lccccc}
\hline & \multicolumn{2}{c}{ Short-CNT-reinforced nanocomposite } & & \multicolumn{2}{c}{ Long-CNT-reinforced nanocomposite } \\
\cline { 2 - 3 } Elastic constants $(\mathrm{GPa})$ & Perfect bonding & Imperfect bonding & & Perfect bonding & Imperfect bonding \\
\hline$E$ & 57.1822 & $51.4138(-10.08)^{*}$ & & 64.6052 & $57.9400(-10.31)$ \\
$G$ & 21.2320 & $18.9496(-10.75)$ & & 23.7620 & $21.1277(-11.08)$ \\
\hline
\end{tabular}

* Percentage loss in elastic properties of imperfectly bonded nanocomposite as compared to perfectly bonded nanocomposite. 


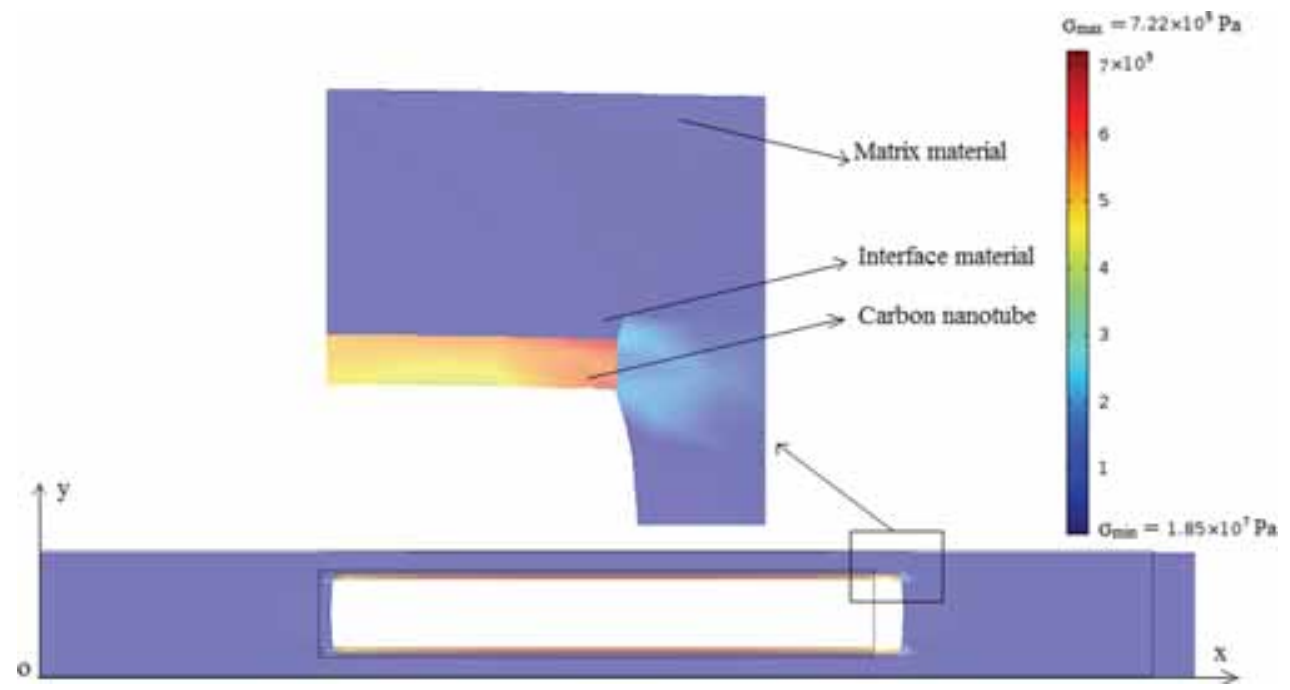

Figure 4. von-Mises stress plot of CNT-Mg nanocomposite considering the effect of interface, for the volume fraction of $4 \%$ at $z=a /$ 2.

bonding, which would result in the loss of stiffness property of CNT-Mg nanocomposite. This finding on effect of interface on the elastic properties of nanocomposite is in good concurrence with the results reported by Joshi and Upadhyay [10].

It can also be observed from table 3 that long-CNT-reinforced nanocomposite possesses higher elastic moduli than those of short-CNT nanocomposite for perfect as well as imperfect bonding, thus establishing the fact that long CNTs are found to be better reinforcement as compared with short CNTs.

\subsection{Post-buckling behaviour of the nanocomposite plate}

The elastic properties of CNT-Mg nanocomposite obtained from the above-mentioned study are utilized to predict the postbuckling behaviour of the resulting nanocomposite plate and to study the effect of CNT reinforcement on the post-buckling behaviour of nanocomposite plate along with the interphase between CNT and matrix material. A full square plate of side (i.e., b) $279 \mathrm{~mm}$ (as used by Kumar and Singh [44]) and of thickness $5.58 \mathrm{~mm}$ (to keep width-to-thickness ratio equal to 50 for a thin plate) is taken to carry out the study.

Five types of flexural boundary conditions, namely SSSS, SSSC, SCSC, SCCC and CCCC, are considered for the study purpose. SSSS refers to a plate with all edges simply supported, SSSC refers to a plate with three edges (i.e., $x=b, y=0$ and $y=b$ ) simply supported and one edge (i.e., $x=0$ ) clamped, SCSC refers to a plate with two longitudinal edges $(y=0$ and $y=b)$ simply supported and other two edges (i.e., $x=0$ and $x=b$ ) clamped, SCCC refers to one edge (i.e., $y=0$ ) simply supported and all other edges clamped and CCCC refers to a plate with all edges clamped. In all above-mentioned boundary conditions, the in-plane boundary conditions on edges $x=0$, $x=b, y=0$ and $y=b$ (as depicted in figure 5) related to in-plane displacements in $x$ and $y$ directions (i.e., $u$ and $v$, respectively) are identical, and uni-axial compression load per unit width is applied on the edge $x=b$. It is to be mentioned that, except for the study on effect of boundary condition on post-buckling behaviour of nanocomposite plate, all other studies are made for all edges simply supported (i.e., SSSS boundary condition).

5.2a Finite-element procedure for post-buckling analysis of nanocomposite plate: To carry out the present study on post-buckling behaviour of the nanocomposite plate, a special purpose program based on finite-element formulation was developed. The formulation is based on first-order shear deformation theory in conjunction with geometrical non-linearity as per von Karman's assumptions. A ninenoded Lagrangian element with $C^{0}$ continuity having five degrees of freedom per node (i.e., $u, v, w, \theta_{x}$ and $\theta_{y}$ ) is used to discretize the plate. The resulting non-linear algebraic equations are solved by the Newton-Raphson technique. The in-depth details of non-linear finite-element formulation of a plate for the post-buckling analysis can be found elsewhere (see Reddy [45, 46]).

5.2b Validation: To verify the results of current formulation for FEM-based study on post-buckling behaviour of nanocomposite, an isotropic, simply supported square plate subjected to uni-axial edge compression, as shown in figure 5 , is considered for comparing the buckling and postbuckling plot with those reported by Sundaresan et al [47] and Le-manh and Lee [48]. The material properties and width-thickness ratio taken for comparison purpose are the same as used by Sundaresan et al [47] and Le-manh and Lee [48], and given below: 


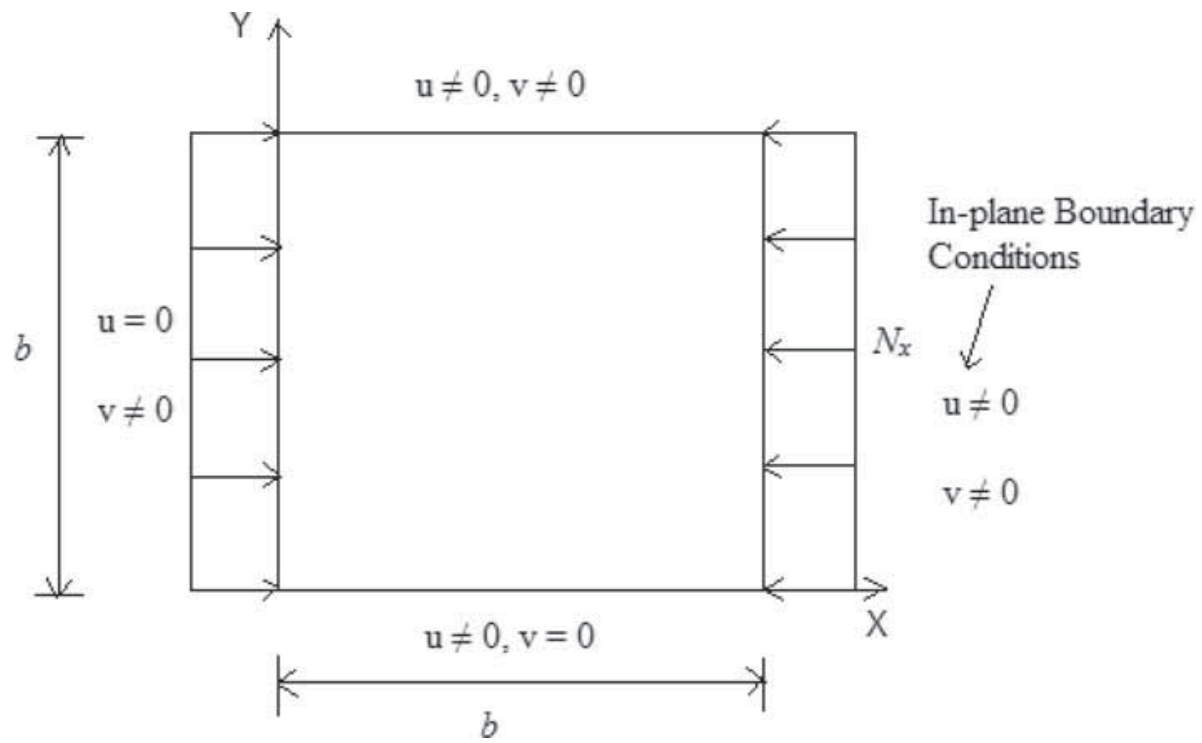

Figure 5. In-plane boundary conditions for a square plate.

$$
E=3 \times 10^{6} \text { psi, } v=0.25, b / h=50 .
$$

While comparing the results, the applied uni-axial edge compressive load $N_{x}$ and the corresponding maximum transverse deflection of plate $w$ are normalized as follows:

$$
\lambda=\frac{N_{x} b^{2}}{\pi^{2} D} \text { and } \overline{\mathrm{w}}=\frac{w}{h}
$$

where $D$, flexural rigidity of the plate, is given by $\frac{E h^{3}}{12\left(1-v^{2}\right)}$, $b$ is the side of the square plate and $h$ represents thickness of the plate.

A good agreement of buckling and post-buckling plot obtained in the present study with that reported by Sundaresan et al [47] and Le-manh and Lee [48] can be seen in figure 6. A little deviation in the plots in figure 6 can be attributed to the different elements used, also commented upon by Le-manh and Lee [48]. Sundaresan et al [47] made use of an eight-noded isoparametric plate finite element with five degrees of freedom per node and Le-manh and Lee [48] used NURBS-based iso-geometric elements with higher order continuity (i.e., $C^{1}$ ), whereas nine-noded Lagrangian elements (having $C^{0}$ continuity) with five degrees of freedom per node are used in the present work.

5.2c Results and discussion: In this section, results corresponding to the post-buckling behaviour of an $\mathrm{Mg}$ plate reinforced with randomly oriented CNTs under in-plane compressive load are presented and discussed. Stiffness properties of the nanocomposite are evaluated for $4 \%$ volume fraction of CNT reinforcement with and without the effect of $\mathrm{vdW}$ bonding between nanotube and matrix material. A parametric study has been conducted to investigate the effects of interphase between CNT and matrix,

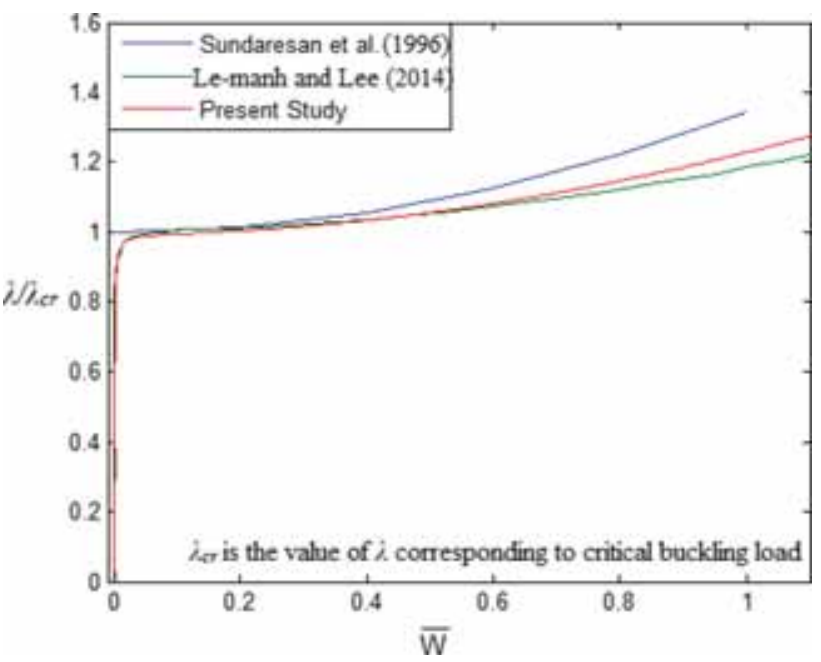

Figure 6. Validation of post-buckling behaviour of simply supported isotropic plate under uni-axial compression with Lemanh and Lee [48] and Sundaresan et al [47].

short-CNT and long-CNT reinforcements in nanocomposite and boundary conditions on buckling and post-buckling response of nanocomposite plate.

The effect of interphase (perfect and imperfect) between $\mathrm{CNT}$ and $\mathrm{Mg}$ matrix on the post-buckling response of simply supported nanocomposite plate reinforced with short-CNT reinforcement is shown in figure 7 . It can be seen from figure 8 that the plate having perfect bonding between CNT and Mg matrix has the highest buckling load and post-buckling strength corresponding to a particular value of transverse deflection, and in deep post-buckling region, this plate becomes stiffer at large deflection than that at initial post-buckling stage. Perfectly bonded CNT- 


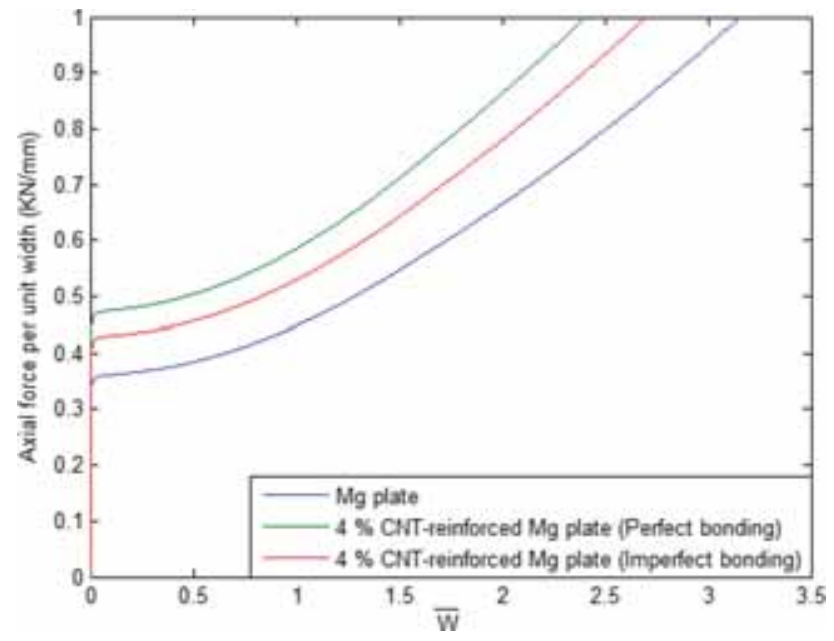

Figure 7. Comparative post-buckling behaviour of magnesium $(\mathrm{Mg})$ plate and CNT-Mg nanocomposite plate having 4\% shortCNT reinforcement with perfect and imperfect bondings, for SSSS boundary condition.

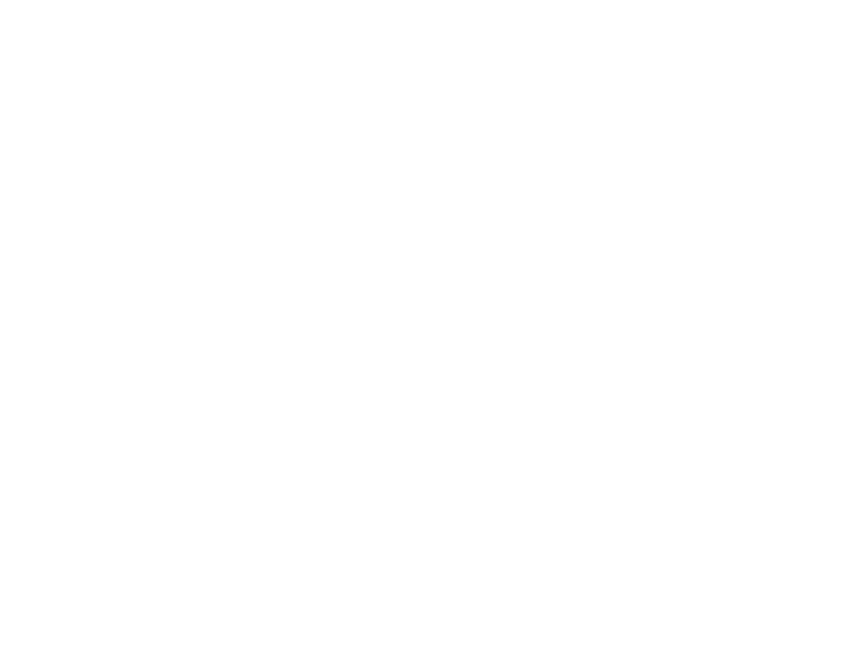

Figure 8. Effect of CNT length on the post-buckling behaviour of simply supported CNT-Mg plate considering the effect of type of bonding between $\mathrm{CNT}$ and $\mathrm{Mg}$.

reinforced plates have approximately $30 \%$ more buckling strength than pure Mg plates, whereas this improvement in buckling strength is $19 \%$ while considering the interphase between CNT and Mg matrix material. This reduced enhancement in the buckling strength can be attributed to the reduced enhancement in stiffness properties of nanocomposite because of weak interphase between CNT and matrix material that would result in poor load transfer to the CNT in the case of imperfect bonding. This is in good concurrence with the findings reported by Kumar and Srinivas [38] and Joshi and Upadhyay [10].

Figure 8 shows the effect of length of CNT on the buckling and post-buckling behaviour of a simply supported (i.e., SSSS) nanocomposite plate with perfect and imperfect

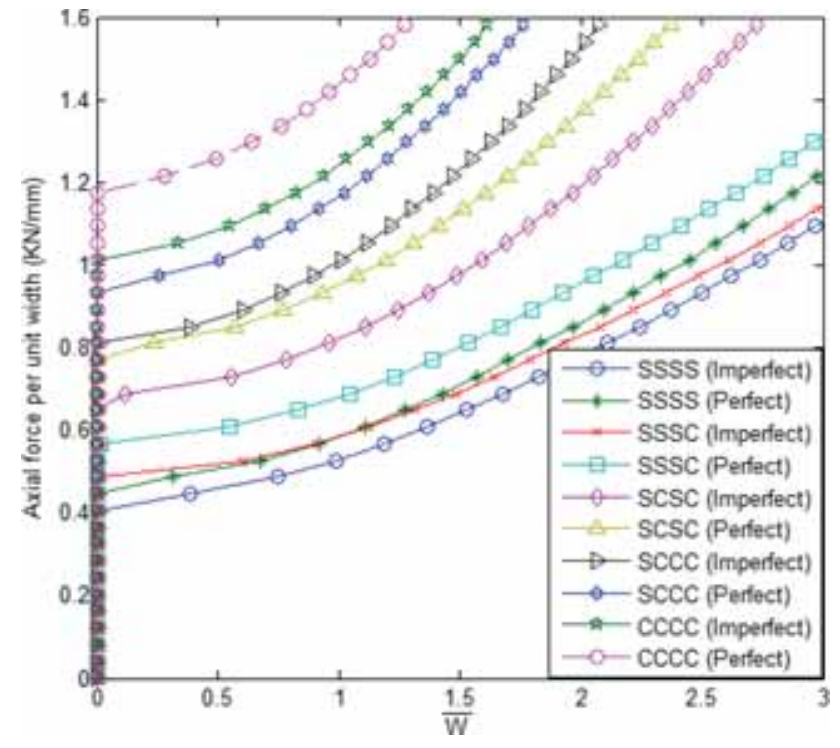

Figure 9. Comparative post-buckling behaviour of $4 \%$ volume fraction of short-CNT-Mg nanocomposite considering different BCs with the effect of vdW interaction between CNT and Mg.

bonding between CNT and matrix material. It can be noticed from figure 8 that the buckling load as well as post-buckling strength at a particular value of transverse deflection is higher for long-CNT-reinforced nanocomposite plate than that of short-CNT-reinforced plate, irrespective of bonding between CNT and matrix material. Further, the buckling strengths of perfectly bonded long- and short-CNT-reinforced nanocomposite plates are found to be nearly $50 \%$ and $30 \%$, respectively, more than that of pure $\mathrm{Mg}$ plate, but because of introduction of interface between CNT and Mg matrix, this enhancement is reduced to $36 \%$ and $19 \%$ for long- and shortCNT nanocomposites, respectively. Considering the effect of interphase, if the comparison is made of the enhancement of buckling strength of long-CNT-reinforced nanocomposite plate in comparison with short-CNT-reinforced nanocomposite, it is found to be approximately $13 \%$ more in the case of long-CNT-reinforced nanocomposite plate.

Post-buckling strength at a particular value of transverse deflection of long- and short-CNT nanocomposite plate is also found to be reduced because of imperfect bonding.

The effects of different boundary conditions on the postbuckling behaviour of short-CNT nanocomposite plate (with and without the effect of interphase) are plotted in figure 9. It is observed from figure 9 that boundary conditions have significant effect on buckling and post-buckling response of nanocomposite plates. The buckling load and post-buckling response in initial stages in terms of strength and stiffness at a particular value of transverse deflection of the CNT-Mg nanocomposite plate are enhanced for perfectly as well as imperfectly bonded nanocomposite plates if the edge conditions are successively changed from simply supported to clamped; therefore, a plate with all edges clamped has maximum buckling and post-buckling strength and stiffness. It can 
also be noted from figure 9 that the loss in buckling strength of nanocomposite plate because of imperfect bonding between CNT and matrix material as compared with perfectly bonded CNT and matrix material also increases as the edge conditions are successively changed from simply supported to clamped. For example, the buckling strengths of a nanocomposite plate with perfect and imperfect bonding between CNT and matrix material are found to be 0.7703 and $0.6488 \mathrm{kN} / \mathrm{mm}$, respectively, in the case of SCSC boundary condition, and therefore a loss of $0.1215 \mathrm{kN} / \mathrm{mm}$ in buckling strength of nanocomposite plate is observed because of the interface, whereas the buckling strengths are 1.1753 and $1.0133 \mathrm{KN} / \mathrm{mm}$, respectively, for perfect and imperfect bonding in the case of CCCC boundary condition with a loss of $0.1620 \mathrm{kN} / \mathrm{mm}$ buckling strength. For all of the boundary conditions, as the deflection of the plate increases, the plate becomes stiffer in deep postbuckling region than in initial post-buckling stage, as shown in figure 9.

\section{Concluding remarks}

Bucking and post-buckling behaviour of a nanocomposite plate reinforced with randomly oriented CNTs has been presented, including the effect of type of bonding (i.e., perfect or imperfect) between $\mathrm{CNT}$ reinforcement and $\mathrm{Mg}$ matrix. The elastic behaviour of interphase region between CNT and $\mathrm{Mg}$ matrix is described by the cohesive zone model given by Jiang et al [21] and then an RVE, with and without interface between CNT and matrix, is analysed to predict the resultant stiffness properties of the nanocomposite. The obtained elastic properties of CNT-Mg nanocomposite are utilized to further study the effect of CNT reinforcement on the buckling and post-buckling behaviour of nanocomposite plate. A special purpose program based on finite-element formulation is developed to study the post-buckling behaviour of nanocomposite plate. The formulation is based on the first-order shear deformation theory in conjunction with geometrical non-linearity as per von Karman's assumptions. The resulting nonlinear algebraic equations are solved by the Newton-Raphson technique. A parametric study has been conducted to investigate the effects of interphase between CNT and matrix, short-CNT and long-CNT reinforcements in nanocomposite and boundary conditions on buckling and post-buckling response of nanocomposite plate. Based on the study conducted, following important conclusions are drawn:

- Imperfect bonding between CNT and Mg results in the loss of buckling and post-buckling strength of the nanocomposite plate as compared with perfectly bonded CNT-Mg nanocomposite plate.

- Buckling load and post-buckling strength at a particular value of transverse deflection are higher for longCNT-reinforced nanocomposite plate than that of short-CNT-reinforced plate, irrespective of bonding between CNT and matrix material.
- The buckling load and post-buckling response in the initial stages in terms of strength and stiffness at a particular value of transverse deflection of the CNT$\mathrm{Mg}$ nanocomposite plate are enhanced for perfectly as well as imperfectly bonded nanocomposite plates, if the edge conditions are successively changed from simply supported to clamped.

\section{Nomenclature}

$\phi$

$\sigma$

$\varepsilon$

$r$

$\rho_{\mathrm{C}}$

$\rho_{m}$

$h_{0}$

E

S

a

$L$

$\sigma_{i j}, \varepsilon_{k l}$ and

$C_{i j k l}$

$\overline{\sigma_{i j}}$ and $\overline{\varepsilon_{k l}}$

$C_{i j k l}^{e}$

$E_{1}$

$E_{2}$

$V_{R V E}$

$v_{f}$

$r_{o}$ and $r_{i}$

$t$

$r_{I}$

b

$h$

$u, v$ and $w$

$\theta_{x}$ and $\theta_{y}$

$\bar{W}$

$N_{x}$ cohesive energy

van der Waals radius

bond energy at equilibrium distance

distance between the particles

area density of CNT

volume density of matrix material

equilibrium distance between the nanofiller

and matrix material

elastic modulus

strain

side of square RVE

length of RVE

stress, strain and stiffness tensor, respectively

volumetric average of stress and strain of RVE

effective stiffness tensor

Young's modulus in the axial direction of nanocomposite

Young's modulus in the transverse direction of nanocomposite

volume of represented volume element

volume fraction for CNT

external and internal radius of the CNT, respectively

thickness of CNT

outer radius of interphase zone

width of square plate

thickness of square plate

displacements in $x, y$ and $z$ directions,

respectively

rotation of normal to the undeformed mid-

plane in $x z$ - and $y z$-plane, respectively

normalized maximum transverse deflection

uni-axial edge compressive load

\section{References}

[1] Esawi A M K and Farag M M 2007 Carbon nanotube reinforced composites: potential and current challenges. Mater. Des. 28: 2394-2401

[2] Iijima S 1991 Helical microtubules of graphitic carbon. Nature 354: 56-58 
[3] Salvetat J P, Bonard J M, Thomson N H, et al 1999 Mechanical properties of carbon nanotubes. Appl. Phys. A Mater. Sci. Process. 69: 255-260

[4] Fiedler B, Gojny F H, Wichmann M H G, et al 2006 Fundamental aspects of nano-reinforced composites. Compos. Sci. Technol. 66: 3115-3125

[5] Esawi A M K, Morsi K, Sayed A, et al 2010 Effect of carbon nanotube $(\mathrm{CNT})$ content on the mechanical properties of CNT-reinforced aluminium composites. Compos. Sci. Technol. 70: 2237-2241

[6] Bakshi S R and Agarwal A 2010 An analysis of the factors affecting strengthening in carbon nanotube reinforced aluminum composites. Carbon 49: 533-544

[7] Yang M, Koutsos V and Zaiser M 2005 Interactions between polymers and carbon nanotubes: a molecular dynamics study. J. Phys. Chem. B 109: 10009-10014

[8] Zhang Y, Zhuang X, Muthu J, et al 2014 Load transfer of graphene/carbon nanotube/polyethylene hybrid nanocomposite by molecular dynamics simulation. Composites Part B 63: 27-33

[9] Choi J, Shin H and Cho M 2016 A multiscale mechanical model for the effective interphase of SWNT/epoxy nanocomposite. Polymer 89: 159-171

[10] Joshi P and Upadhyay S H 2014a Effect of interphase on elastic behavior of multiwalled carbon nanotube reinforced composite. Comput. Mater. Sci. 87: 267-273

[11] Liu Y J and Chen X L 2003a Evaluations of the effective material properties of carbon nanotube-based composites using a nanoscale representative volume element. Mech. Mater. 35: 69-81

[12] Liu Y J and Chen X L 2003b Continuum models of carbon nanotube-based composites using the boundary element method. Electron. J. Bound. Elem. 1: 316-335

[13] Joshi U A, Sharma S C and Harsha S P 2011 Effect of waviness on the mechanical properties of carbon nanotube based composites. Phys. E. Low-dim. Syst. Nanostruct. 43:1453-1460

[14] Shokrieh M M and Rafiee R 2010a On the tensile behavior of an embedded carbon nanotube in polymer matrix with nonbonded interphase region. Compos. Struct. 92: 647-652

[15] Joshi P and Upadhyay S H 2014b Evaluation of elastic properties of multi-walled carbon nanotube reinforced composite. Comput. Mater. Sci. 81: 332-338

[16] Tsai J L, Tzeng S H and Chiu Y T 2010 Characterizing elastic properties of carbon nanotubes/polyimide nanocomposites using multi-scale simulation. Composites Part B 41: 106-115

[17] Herasati S, Zhang L C and Ruan H H 2014 A new method for characterizing the interphase regions of carbon nanotube composites. Int. J. Solids Struct. 51: 1781-1791

[18] Shokrieh M M and Rafiee R 2010b Prediction of mechanical properties of an embedded carbon nanotube in polymer matrix based on developing an equivalent long fiber. Mech. Res. Commun. 37: 235-240

[19] Rafiee R and Pourazizi R 2015 Influence of CNT functionalization on the interphase region between CNT and polymer. Comput. Mater. Sci. 96: 573-578

[20] Odegard G M, Clancy T C and Gates T S 2005 Modeling of the mechanical properties of nanoparticle/polymer composites. Polymer 46: 553-562
[21] Jiang L Y, Huang Y, Jiang H, et al 2006 A cohesive law for carbon nanotube/polymer interfaces based on the van der Waals force. J. Mech. Phys. Solids 54: 2436-2452

[22] Tan H, Jiang L Y, Huang Y, et al 2007 The effect of van der Waals-based interface cohesive law on carbon nanotube-reinforced composite materials. Compos. Sci. Technol. 67: 2941-2946

[23] Zhao J, Jiang J W, Jia Y, et al 2013 A theoretical analysis of cohesive energy between carbon nanotubes, graphene and substrates. Carbon 57: 108-119

[24] Zhang Y, Zhao J, Jia Y, et al 2013 An analytical solution on interface debonding for large diameter carbon nanotube-reinforced composite with functionally graded variation interphase. Compos. Struct. 104: 261-269

[25] Arani A G, Maghamikia S, Mohammadimehr M and Arefmanesh A 2011 Buckling analysis of laminated composite rectangular plates reinforced by SWCNTs using analytical and finite element methods. J. Mech. Sci. Technol. 25: 809-820

[26] Jafari Mehrabadi S, Sobhani Aragh B, Khoshkhahesh V, Taherpour A 2012 Mechanical buckling of nanocomposite rectangular plate reinforced by aligned and straight singlewalled carbon nanotubes. Compos Part B Eng 43: 2031-2040

[27] Lei Z X, Liew K M, Yu J L 2013 Buckling analysis of functionally graded carbon nanotube-reinforced composite plates using the element-free kp-Ritz method. Compos Struct 98: $160-168$

[28] Shen H S and Zhang C L 2010 Thermal buckling and postbuckling behavior of functionally graded carbon nanotubereinforced composite plates. Mater. Des. 31: 3403-3411

[29] Shen H S and Zhu Z H 2012 Postbuckling of sandwich plates with nanotube-reinforced composite face sheets resting on elastic foundations. Eur. J. Mech. A/Solids 35: 10-21

[30] Boda D and Henderson D 2008 The effects of deviations from Lorentz-Berthelot rules on the properties of a simple mixture. Mol. Phys. Int. J. Interface Between Chem. Phys. 106: 2367-2370

[31] White A 2000 Intermolecular potentials of mixed systems: testing the Lorentz-Berthelot mixing rules with ab initio calculations. DSTO-TN-0302

[32] Sears A and Batra R C 2004 Macroscopic properties of carbon nanotubes from molecular-mechanics simulations. Phys. Rev. B 69: 235406

[33] Sun C T, Vaidya R S 1996 Prediction of composite properties from a representative volume element. Compos Sci Technol 56: 171-179

[34] Tsai S W and Pagano N J 1968 Invariant properties of composite materials. In: Composite Materials Workshop Proceedings. Technomic Publishing Co.

[35] Kunanopparat T, Menut P, Morel M H and Guilbert S 2008 Plasticized wheat gluten reinforcement with natural fibers: effect of thermal treatment on the fiber/matrix adhesion. Compos. Part A Appl. Sci. Manuf. 39: 1787-1792

[36] Stern T, Teishev A and Marom G 1997 Composites of polyethylene reinforced with chopped polyethylene fibers: effect of transcrystalline interphase. Compos. Sci. Technol. 57: $1009-1015$

[37] Joshi U A, Joshi P, Harsha S P and Sharma S C 2010 Evaluation of the mechanical properties of CNT based 
composites using hexagonal RVE. J. Nanotechnol. Eng. Med. 1: 1-7

[38] Kumar P and Srinivas J 2014 Numerical evaluation of effective elastic properties of CNT-reinforced polymers for interphase effects. Comput. Mater. Sci. 88: 139-144

[39] Tucker C L and Liang E 1999 Stiffness predictions for unidirectional short-fiber composites: review and evaluation. Compos. Sci. Technol. 59: 655-671

[40] Hu Z, Arefin M R H, Yan X and Fan Q H 2014 Mechanical property characterization of carbon nanotube modified polymeric nanocomposites by computer modeling. Compos. Part B Eng. 56: 100-108

[41] Fan J 2011 Mutiscale analysis of deformation and failure of materials. John Wiley \& Sons Ltd, New York.

[42] Bentor Y 2014. http://www.chemicalelements.com/index. html. Accessed 5 Jan 2016

[43] Lu W B, Wu J, Song J, et al 2008 A cohesive law for interfaces between multi-wall carbon nanotubes and polymers due to the van der Waals interactions. Comput. Methods Appl. Mech. Eng. 197: 3261-3267

[44] Kumar D and Singh S B 2010 Effects of boundary conditions on buckling and postbuckling responses of composite laminate with various shaped cutouts. Compos. Struct. 92: 769-779

[45] Reddy J N 2004a Mechanics of laminated composite plates and shells, second ed. CRC Press, Boca Raton

[46] Reddy J N 2004b An introduction to nonlinear finite element analysis. Oxford University Press, Oxford

[47] Sundaresan P, Singh G and Venkateswara Rao G 1996 Buckling and post-buckling analysis of moderately thick laminated rectangular plates. Comput. Struct. 61: 79-86

[48] Le-manh T and Lee J 2014 Postbuckling of laminated composite plates using NURBS-based isogeometric analysis. Compos. Struct. 109: 286-293 